\title{
ARTICLE
}

Clinical Study

\section{Imatinib in combination with phosphoinositol kinase inhibitor buparlisib in patients with gastrointestinal stromal tumour who failed prior therapy with imatinib and sunitinib: a Phase $1 \mathrm{~b}$, multicentre study}

\author{
Hans Gelderblom ${ }^{1}$, Robin L. Jones ${ }^{2}$, Suzanne George ${ }^{3}$, Claudia Valverde Morales ${ }^{4}$, Charlotte Benson ${ }^{5}$, Jean-Yves Blay ${ }^{6,7}$, \\ Daniel J. Renouf ${ }^{8}$, Toshihiko Doi ${ }^{9}$, Axel Le Cesne ${ }^{10}$, Michael Leahy ${ }^{11}$, Sabine Hertle ${ }^{12}$, Paola Aimone ${ }^{12}$, Ulrike Brandt ${ }^{12}$ and \\ Patrick Schöffski ${ }^{13}$
}

BACKGROUND: The majority of patients with advanced gastrointestinal stromal tumours (GISTs) develop resistance to imatinib and sunitinib, the standard of care for these patients. This study evaluated the combination of buparlisib, an oral phosphoinositide 3-kinase (PI3K) inhibitor, with imatinib in patients with advanced GIST, who have failed prior therapy with imatinib and sunitinib.

METHODS: This Phase 1b, multicentre, open-label study aimed to determine the maximum tolerated dose (MTD) and/or a recommended Phase 2 dose of buparlisib in combination with $400 \mathrm{mg}$ of imatinib through a dose-escalation part and a doseexpansion part, and also evaluated the clinical profile of the combination.

RESULTS: Sixty patients were enrolled, including 25 in the dose-escalation part and 35 in the dose-expansion part. In the combination, MTD of buparlisib was established as $80 \mathrm{mg}$. No partial or complete responses were observed. The estimated median progression-free survival was 3.5 months in the expansion phase. Overall, $98.3 \%$ of patients had treatment-related adverse events (AEs), including $45 \%$ with grade 3 or $4 \mathrm{AEs}$.

CONCLUSIONS: Buparlisib in combination with imatinib provided no additional benefit compared with currently available therapies. Due to the lack of objective responses, further development of this combination was not pursued for third-line/ fourth-line advanced/metastatic GIST.

TRIAL REGISTRATION NUMBER: NCT01468688.

British Journal of Cancer (2020) 122:1158-1165; https://doi.org/10.1038/s41416-020-0769-y

\section{BACKGROUND}

Gastrointestinal stromal tumours (GISTs) are rare tumours of mesenchymal origin, most likely arising from precursors of the interstitial cells of Cajal of the gastrointestinal tract. ${ }^{1}$ The estimated incidence of GIST is $12-15$ cases per 1 million population in Western countries. ${ }^{2,3}$ Approximately $75-80 \%$ of GIST cases show activating mutations in KIT (CD117). The most common KIT mutations are found in exon 11 in $70 \%$ of cases, while KIT exon 9 mutations are relatively less common and are found in $10-12 \%$ of GIST cases. Mutations in KIT exons 13 and 17 are rare. ${ }^{4}$ A subset (5-8\%) of GIST harbours PDGFRA mutations without the KIT mutations. ${ }^{5,6}$ The first-line treatment for patients with primary
GIST is surgical resection. However, with surgery alone, the recurrence rates are more than $50 \%$ within 2 years, and the 5 -year disease-specific survival rate is about 54\%. ${ }^{7}$

Imatinib (Gleevec ${ }^{\circledR} /$ Glivec $^{\circledR}$, Novartis), a tyrosine kinase inhibitor (TKI), is approved for the treatment of adult patients with $K \Gamma^{+}$ (CD117) unresectable/metastatic GIST, and as adjuvant treatment following resection of high-risk GIST. ${ }^{8,9}$ For patients who develop either primary or secondary resistance to imatinib due to mutations in KIT or PDGFRA, sunitinib, a TKI-targeting multiple tyrosine kinase, demonstrated a significant improvement in progression-free survival (PFS) ${ }_{10}^{10}$ and is globally approved for the treatment of metastatic GIST. ${ }^{11}$ Following the failure of imatinib

${ }^{1}$ Leiden University Medical Center, Leiden, The Netherlands; ${ }^{2}$ The Royal Marsden Hospital and Institute of Cancer Research, London, UK; ${ }^{3}$ Dana-Farber Cancer Institute

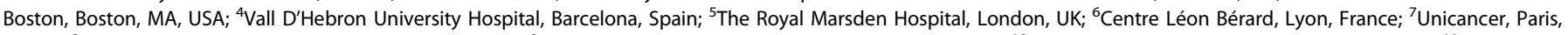

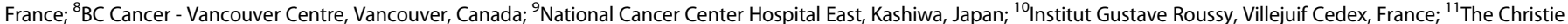

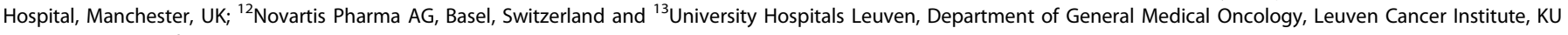
Leuven, Leuven, Belgium

Correspondence: Hans Gelderblom (a.j.gelderblom@lumc.nl)

Previous affiliation for Robin L. Jones: Fred Hutch Cancer Research Center, University of Washington, Seattle, WA, USA

Received: 4 September 2019 Accepted: 12 February 2020

Published online: 9 March 2020 
and sunitinib, treatment with regorafenib, another TKI targeting multiple oncogenic pathways, showed a significant improvement in PFS when compared with placebo, and was approved as a thirdline therapy in 2013.9,12,13 In addition, reintroduction of imatinib was found to be marginally beneficial in patients with metastatic GIST in delaying disease progression. ${ }^{14,15}$ Despite the availability of current treatment options, there still remains a high unmet medical need for patients with advanced/metastatic GIST.

Advanced GISTs are commonly associated with resistance against approved TKIs, perhaps due to the gain of TKI-resistant mutations in $K I T .^{16}$ In addition, resistance might be the result of either genomic amplification of KIT or activation of alternative oncogenic signalling mechanisms, including the phosphoinositide 3-kinase (PI3K)/protein kinase B (AKT)/mammalian target of rapamycin (mTOR) pathway. ${ }^{16}$ The PI3K/AKT signalling pathway has also been shown to be activated in a KIT-dependent manner in primary GISTs, specifically with mutations in exons 9, 11 and $13 .{ }^{17}$ Buparlisib (BKM120), an oral PI3K inhibitor, has shown antitumour activity against both imatinibsensitive and imatinib-resistant cell lines with activated PI3K pathway. Buparlisib in combination with imatinib, has shown a greater suppression of tumour growth compared with single-agent imatinib or buparlisib. In patient-derived GIST xenograft models with imatinib-resistant cell lines, similar results were observed, with a significant inhibition of the tumour growth with the buparlisib and imatinib combination compared with single agents. ${ }^{18-20}$

It was thus hypothesised that the inhibition of PI3K/AKT signalling pathways by buparlisib in addition to continuous inhibition of KIT/PDGFRA mutations by imatinib could lead to increased clinical efficacy, and possibly overcome the acquired resistance to imatinib. This Phase $1 \mathrm{~b}$ study assessed the maximum tolerated dose (MTD), and the safety and efficacy of the combination of buparlisib and imatinib in patients with advanced GIST, who had failed both imatinib and sunitinib.

\section{METHODS}

Patients

Adult patients with histologically confirmed unresectable or metastatic GIST who had failed prior therapy with both imatinib and sunitinib were enrolled. Patients were required to have at least one measurable lesion as defined by Response Evaluation Criteria in Solid Tumors (RECIST, version 1.1), a World Health Organisation performance status of $0-2$ and adequate bone marrow and organ function. For the dose-expansion part, radiological confirmation of disease progression during previous therapy was required, and up to three lines of prior therapy were permitted. Patients with previous treatment with $\mathrm{PI} 3 \mathrm{~K}$ inhibitors, any ongoing condition of Common Terminology Criteria for Adverse Events (CTCAE) grade $\geq 2$ related to previous imatinib and/or sunitinib and poorly controlled diabetes mellitus (defined as glycosylated haemoglobin $>8 \%$ ), were excluded from the study. Since buparlisib is known to be associated with psychiatric disorders, including mood disorders, anxiety and depression, patients with a medically documented history of active major psychiatric disorders were not eligible. At screening, patients who had a cut-off score of $\geq 10$ in the 9-item depression scale of the Patient Health Questionnaire-9 (PHQ-9) or a cut-off score of $\geq 15$ in the Generalized Anxiety Disorder Assessment-7 (GAD-7) mood scale, or selected a positive response to question numbers 1,2 and 3 to question number 9 regarding potential for suicidal thoughts or ideation in the PHQ-9 (independent of the total score of the PHQ-9) were excluded from the study. ${ }^{21,22}$

Study design and treatment

This was a multicentre, open-label, Phase $1 \mathrm{~b}$ study to investigate the safety and tolerability of escalating doses of buparlisib in combination with imatinib in patients with metastatic/unresectable GIST. The primary end point was to determine the MTD and/or a recommended Phase 2 dose (RP2D) of buparlisib with $400 \mathrm{mg}$ of imatinib. The key secondary end points were to assess the safety, tolerability and clinical activity of the combination.

The study was conducted in two stages: a dose-escalation part to establish the MTD and/or RP2D and a dose-expansion part at the achieved MTD/RP2D. In the dose-escalation part, successive cohorts of at least three patients received increasing doses of buparlisib. The initial dose was $40 \mathrm{mg}$ once daily, which was $40 \%$ of $100 \mathrm{mg} /$ day (MTD as monotherapy), followed by 50, 70, 80 and $100 \mathrm{mg}$ of daily doses along with imatinib, $400 \mathrm{mg}$ daily, until the determination of MTD and/or RP2D of buparlisib. At least six patients must have been treated with the specific dose prior to declaring it as MTD. In the dose-expansion part, patients received either imatinib (arm 1) or buparlisib (arm 2) from days 1 to 8 (monotherapy run-in phase), followed by the combination therapy on day 9 , or patients were treated with the combination therapy starting on day 1 (arm 3). All patients were treated until disease progression or early discontinuation.

This study was reviewed and approved by the independent ethics committee and/or local review board at each participating institution, and conducted according to the ethical principles described in the Declaration of Helsinki. All participants provided written informed consent prior to the study.

\section{Assessments}

Efficacy. The tumour response was determined locally by the investigator at each site according to RECIST version 1.1. The efficacy assessments included clinical benefit rate (CBR), overall response rate (ORR), disease control rate (DCR) and PFS. The CBR was defined as the proportion of patients with the best overall response of complete response (CR) or partial response (PR), or a response of stable disease (SD) that lasted for $\geq 16$ weeks after treatment initiation; ORR was defined as the proportion of patients with the best overall response of $C R$ or PR. The DCR was the proportion of patients with the best overall response of CR or PR or SD. The PFS was defined as the time from the date of the first study treatment to the date of the first documented disease progression or date of death due to any cause, whichever occurred first. The response was assessed using computed tomography or magnetic resonance imaging at screening on day 1 of cycle 3 , on day 1 of every second cycle through cycle 11, every 3 months after cycle 12 and every 6 months after cycle 24. The same technique, whether computed tomography or magnetic resonance imaging, that was used to assess the response at screening for a patient, was used throughout the study for a particular patient.

Safety. The adverse events (AEs) were coded using the Medical Dictionary for Regulatory Activities (MedDRA) version 19.0, while the severity was graded according to the CTCAE version 4.03. The safety assessments were performed at each visit. Patients were also asked to complete the mood scales for depression (PHQ-9) and anxiety (GAD-7) at screening, twice during cycle 1 and once during each subsequent cycle, including at the end of treatment. From cycle 12, the questionnaires were completed every 3 months, and after cycle 24, every 6 months.

\section{Statistical methods}

The full-analysis set (FAS) and safety set consisted of all patients who received at least one dose of buparlisib or imatinib. The FAS was used for summarising the baseline and demographic characteristics. The efficacy analysis focused on patients enrolled in the dose-expansion part in the FAS. The Kaplan-Meier method was used for estimating PFS. The ORR, DCR and CBR were summarised with their $95 \%$ confidence intervals ( $\mathrm{Cls}$ ) using exact Pearson-Clopper limits. Descriptive statistics were used to summarise AEs for the safety set.

$\mathrm{SAS}^{\circledR}$ version 9.4 was used in all the statistical analyses. 
Table 1. Baseline characteristics and demographics (FAS).

\begin{tabular}{|c|c|c|c|c|c|c|}
\hline Parameters & \multicolumn{5}{|c|}{ Buparlisib daily dose (+imatinib $400 \mathrm{mg}$ ) } & $\begin{array}{l}\text { All patients } \\
n=60\end{array}$ \\
\hline Median (range) & $40.5(30-72)$ & $52.0(44-63)$ & $63.0(56-78)$ & $57.0(28-78)$ & $63.5(42-72)$ & $56.5(28-78)$ \\
\hline \multicolumn{7}{|l|}{ Age category (years), $n(\%)$} \\
\hline \multicolumn{7}{|l|}{ Sex, $n(\%)$} \\
\hline Male & $2(50.0)$ & $2(50.0)$ & $3(100)$ & $27(62.8)$ & $5(83.3)$ & $39(65.0)$ \\
\hline Female & $2(50.0)$ & $2(50.0)$ & 0 & $16(37.2)$ & $1(16.7)$ & $21(35.0)$ \\
\hline \multicolumn{7}{|l|}{ Race, $n$ (\%) } \\
\hline Caucasian & $4(100)$ & $4(100)$ & $3(100)$ & $39(90.7)$ & $6(100)$ & $56(93.3)$ \\
\hline 1 & $4(100)$ & $2(50.0)$ & 0 & $18(41.9)$ & $2(33.3)$ & $26(43.3)$ \\
\hline 2 & 0 & 0 & 0 & $1(2.3)$ & 0 & $1(1.7)$ \\
\hline \multicolumn{7}{|l|}{ Mitotic index (per $50 \mathrm{HPFs})^{\mathrm{a}}$} \\
\hline$\leq 5$ & 0 & $1(25.0)$ & 0 & $9(20.9)$ & $1(16.7)$ & $11(18.3)$ \\
\hline$>5$ to $\leq 10$ & 0 & 0 & 0 & $5(11.6)$ & $1(16.7)$ & $6(10.0)$ \\
\hline$>10$ & 0 & $3(75.0)$ & $1(33.3)$ & $8(18.6)$ & $2(33.3)$ & $14(23.3)$ \\
\hline Missing & $2(50.0)$ & 0 & $2(66.7)$ & $13(30.2)$ & $2(33.3)$ & $19(31.7)$ \\
\hline \multicolumn{7}{|l|}{ Complete gross resection (primary tumour) } \\
\hline No & $2(50.0)$ & 0 & 0 & $10(23.3)$ & 0 & $12(20.0)$ \\
\hline Yes & $2(50.0)$ & $4(100)$ & $3(100)$ & $33(76.7)$ & $6(100)$ & $48(80.0)$ \\
\hline
\end{tabular}

\section{RESULTS}

Patients

A total of 60 patients ( 21 females) were enrolled between April 19, 2012 and May 02, 2014. Of these, 25 and 35 patients were included in the dose-escalation and dose-expansion part, respectively. The baseline characteristics and demographics of patients are described in Table 1. The median age of the patients was 56.5 years; $65 \%$ were male and $93.3 \%$ were Caucasian, and 48 patients $(80 \%)$ had undergone complete gross resection of primary GIST.

Forty-seven patients (78.3\%) discontinued due to progressive disease, $10(16.7 \%)$ due to AEs and $3(5 \%)$ due to patient's choice.

\section{Treatment exposure}

Overall, the median duration of exposure to study treatment was 2.2 months (range, $0.2-36.8$ ); over $50 \%$ of the patients received treatment for more than 2 months, and $20 \%$ of the patients received treatment for more than 6 months. Thirty-nine patients $(65.0 \%)$ had at least 1 interruption or dose change of buparlisib, including 1 patient with dose escalation, and
33 patients (55.0\%) had at least 1 interruption/dose reduction of imatinib.

Maximum tolerated dose

The dose-determining set consisted of 25 patients over 5 doselevel cohorts of buparlisib along with $400 \mathrm{mg}$ of imatinib. In cohort 1 (40 mg of buparlisib, $n=4$ ), none of the patients reported doselimiting toxicities (DLTs); in cohort 2 (50 mg of buparlisib, $n=4$ ), one patient reported a DLT of anaphylaxis. Cohorts $3(70 \mathrm{mg}, n=$ 3 ) and $4(80 \mathrm{mg}$ of buparlisib, $n=4)$ reported no DLTs, and in cohort 5 ( $100 \mathrm{mg}$ of buparlisib, $n=6$ ), three patients experienced DLTs (stomatitis, hyperglycaemia and depression). Consequently, four additional patients were treated at the previously tested dose level ( $80 \mathrm{mg}$ of buparlisib and $400 \mathrm{mg}$ of imatinib), and none of them experienced a DLT. The MTD for buparlisib in combination with $400 \mathrm{mg}$ of imatinib was therefore declared as $80 \mathrm{mg}$.

Efficacy

The analysis of efficacy was performed on the patients in the doseexpansion part $(n=35)$. All patients received $80 \mathrm{mg}$ of buparlisib 
Table 2. The best overall response (FAS, dose-expansion part).

\begin{tabular}{|c|c|c|}
\hline Parameters & $\begin{array}{l}\text { All patients } \\
n=35, \mathrm{n}(\%)\end{array}$ & $95 \% \mathrm{Cl}$ \\
\hline \multicolumn{3}{|l|}{ Best overall response } \\
\hline Complete response (CR) & 0 & - \\
\hline Partial response (PR) & 0 & - \\
\hline Stable disease (SD) & $19(54.3)$ & - \\
\hline Progressive disease (PD) & $14(40.0)$ & - \\
\hline Unknown & $2(5.7)$ & - \\
\hline $\begin{array}{l}\text { Clinical benefit rate }(C B R): C R+P R+S D \geq \\
16 \text { weeks }\end{array}$ & $10(28.6)$ & $14.6-46.3$ \\
\hline Overall response rate (ORR): $C R+P R$ & 0 & $0-10.0$ \\
\hline Disease control rate $(D C R): C R+P R+S D$ & $19(54.3)$ & $36.6-71.2$ \\
\hline
\end{tabular}

Table 3. Analysis of progression-free survival using Kaplan-Meier method (FAS, dose-expansion part).

\begin{tabular}{lc}
\hline & $\begin{array}{l}\text { All patients } \\
n=35\end{array}$ \\
\hline Number of events, $n$ (\%) & $29(82.9)$ \\
Progression & $27(77.1)$ \\
Death & $2(5.7)$ \\
Number of censoring, $\mathrm{n}(\%)$ & $6(17.1)$ \\
Percentiles $(95 \% \mathrm{Cl})$ (months) & \\
25 th & $1.9(1.7-1.9)$ \\
50 th & $3.5(1.9-5.4)$ \\
75 th & $5.5(3.7-11.4)$ \\
$\%$ Event-free probability estimates (95\% Cl) & \\
2 months & $57.4(38.8-72.2)$ \\
4 months & $37.3(20.8-53.8)$ \\
6 months & $22.4(9.4-38.7)$ \\
8 months & $18.6(7.0-34.6)$ \\
11 months & $11.2(2.9-25.8)$ \\
\hline Cl confidence interval, FAS full-analysis set. & \\
\hline
\end{tabular}

in combination with $400 \mathrm{mg}$ of imatinib. A best overall response of SD was observed in 19 patients (54.3\%). None of the patients achieved a CR or PR (Table 2). The DCR was $54.3 \%(95 \% \mathrm{Cl}$, 36.6-71.2), and the CBR was $28.6 \%(95 \% \mathrm{Cl}, 14.6-46.3)$. The estimated median PFS was 3.5 months $(95 \% \mathrm{Cl}, 1.9-5.4)$, and the estimated PFS rate (Table 3) at 6 months was $22.4 \%(95 \% \mathrm{Cl}$, 9.4-38.7).

Safety

All patients had experienced at least one $A E$, and 39 patients (65.0\%) experienced AEs of grade 3 or 4 . The most common allgrade AEs, regardless of the relationship to study treatment in the overall population, were nausea (63.3\%), fatigue (48.3\%), diarrhoea (45.0\%), decreased appetite, hyperglycaemia (28.3\% each), abdominal pain and anaemia (25.0\% each). The most common grade 3 or 4 AEs included rash $(6.7 \%)$, diarrhoea, hypophosphataemia, asthenia, rash maculopapular, hypokalaemia and decreased appetite (5.0\% each). Overall, $98.3 \%$ of patients had AEs suspected to be related to the study drug; $45.0 \%$ of those reported grade 3 or 4 AEs. The most common AEs suspected to be drug related (Table 4) were nausea (48.3\%) and fatigue (38.3\%). Thirteen patients $(21.7 \%)$ reported $\mathrm{AEs}$, leading to discontinuation

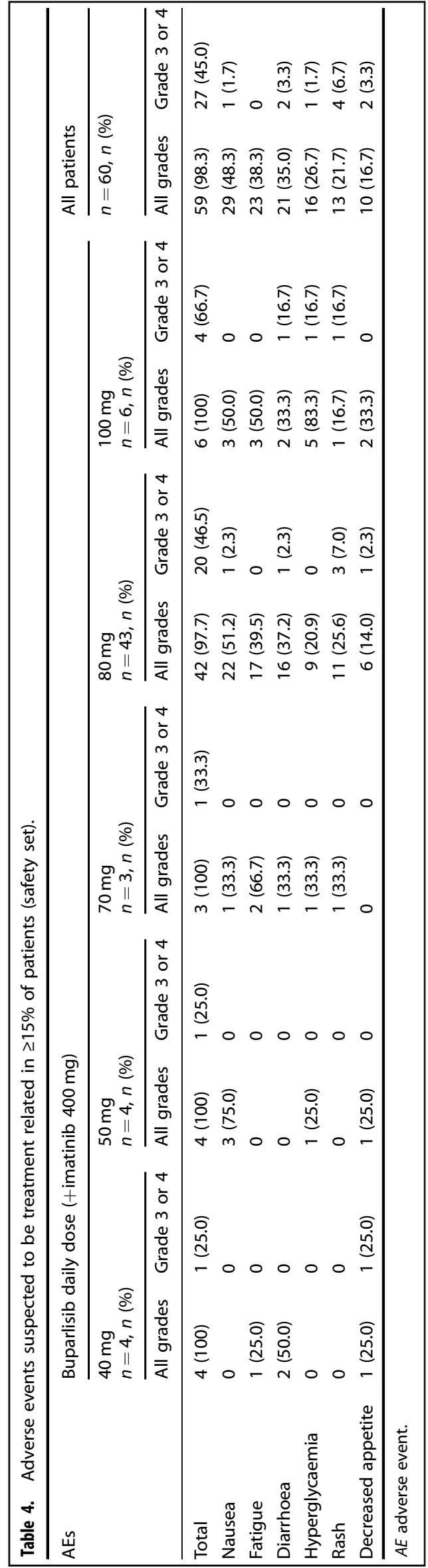


of study treatment (Table 5). In nine patients, these AEs were suspected to be related to the study drug, and in seven patients, these were grade 3 or $4 \mathrm{AEs}$. The main $\mathrm{AE}$ leading to discontinuation was depression (three patients).

\section{Serious adverse events}

In total, 23 patients (38.3\%) experienced serious AEs (SAEs, Table 5). The most common SAEs were abdominal pain, hyponatraemia, nausea and peritoneal haemorrhage in two patients each (3.3\%). Thirteen SAEs were suspected to be drug related in eight patients (13.3\%), and included anaphylactic reaction, hypokalaemia, hypophosphataemia, nausea, dementia, rash maculopapular, posterior reversible encephalopathy syndrome, hyponatraemia, depression, anxiety, delirium, rash and hyperglycaemia. Of these eight patients, four permanently discontinued the study treatment due to SAEs pertaining to depression, anxiety/delirium/rash/hyperglycaemia, posterior reversible encephalopathy syndrome and anaphylactic reaction, respectively.

\section{Neuropsychiatric AEs}

In total, 25 patients (41.7\%) experienced AEs related to psychiatric disorders, of whom, $18(30.0 \%)$ had drug-related AEs. The most common psychiatric disorders regardless of the relationship to study treatment ( $\geq 3 \%$ of patients) were insomnia in $12(20.0 \%)$, anxiety in $9(15.0 \%)$, depression in $8(13.3 \%)$, irritability in $3(5.0 \%)$ and depressed and altered mood in 2 patients each (3.3\%). Three patients (5.0\%) reported grade 3 or 4 AEs of insomnia, depression and hallucination, which were all suspected to be related to the study drug as per the investigator. Four patients reported psychiatric disorders leading to treatment discontinuation; none of them were due to mood disorders. The treatment was temporarily interrupted in seven patients due to psychiatric disorders, and in two patients due to mood disorders (one each for euphoric and depressive mood).

Deaths

Overall, 12 (20.0\%) deaths were reported (Table 5). This included three on-treatment deaths (two due to GIST and one due to infectious meningitis), defined as either death on treatment or death within 30 days of the last dose. Seven patients died after the treatment discontinuation due to GIST, and two patients due to unknown reasons. The on-treatment death due to AEs (meningitis) was not considered to be related to the study treatment by the investigator.

\section{DISCUSSION}

A major challenge in the treatment of GIST is resistance to TKIs, which can be primary resistance, or more commonly due to the acquisition of secondary KIT/PDGFRA mutations or activation of alternative signalling pathways, such as the PI3K/AKT/mTOR pathway. ${ }^{23}$ Another mechanism of resistance to targeted therapies is due to insufficient levels of phosphatase and tensin homologue (PTEN) protein, which negatively regulates the PI3K/ AKT signalling pathway. ${ }^{24}$ The PI3K/AKT pathway plays an important role in the proliferation and survival of imatinibsensitive and -resistant GIST. ${ }^{25}$ Abrogation of the PI3K-binding site in murine models with KIT mutations prevented the development of GIST. ${ }^{26}$ Buparlisib is a pan-PI3K inhibitor that targets all the isoforms of class I PI3K, including PI3Kס, which was hypothesised to play an important role in imatinib-resistant GIST. ${ }^{27}$ One of the most promising approaches is to inhibit the PI3K pathway in addition to continuous inhibition of KIT/PDGFRA mutations by imatinib. This concept had been validated in patient-derived mouse xenografts of GIST. Single-agent buparlisib was able to reduce tumour volume in mice grafted with human GIST carrying diverse KIT genotypes and PTEN genomic status, and a greater reduction was observed in combination with imatinib. ${ }^{20}$ The

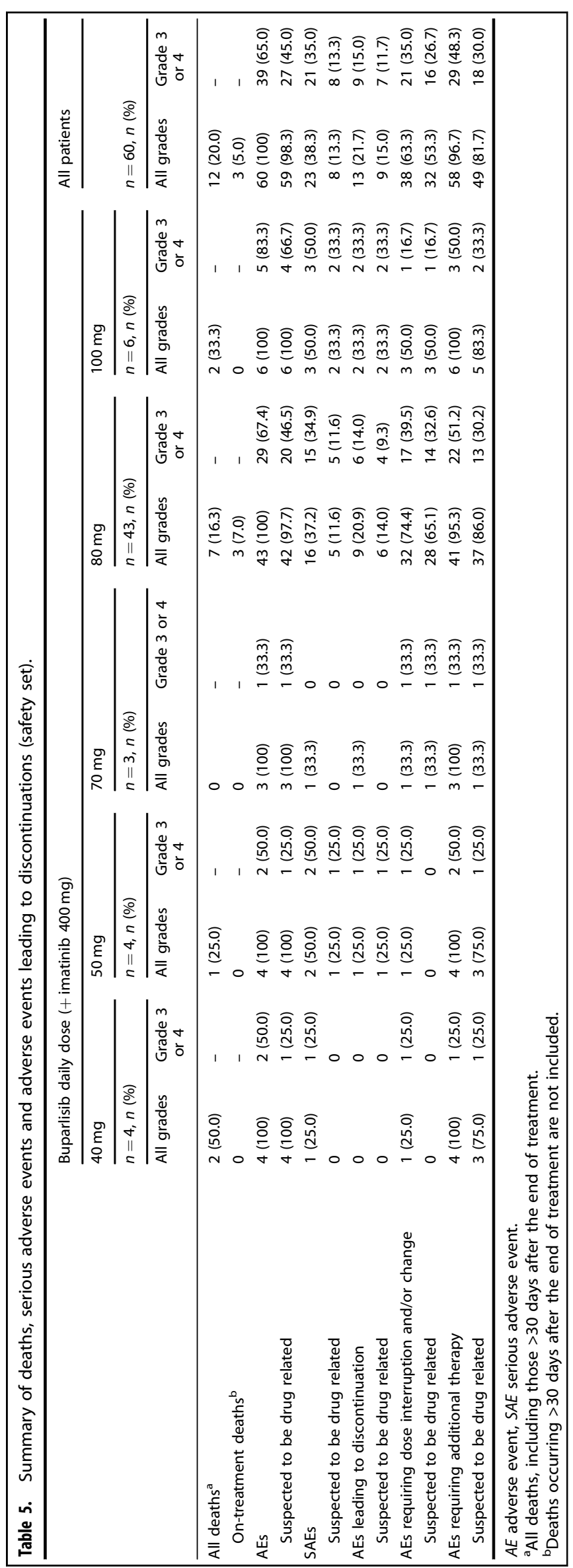


activity of GDC-0941, another PI3K inhibitor, in combination with imatinib, was also reported in a murine model of GIST, with a concurrent loss of the protein and gene expression of PTEN. ${ }^{25}$ In preclinical studies with buparlisib, better efficacy was observed with the combination of GDC-0941 and imatinib compared with single agent alone, with a reduction in tumour growth even after discontinuation of the drug. Similar results were also emulated in preclinical studies with the combination of an AKT inhibitor MK2206 and imatinib in GIST xenografts, ${ }^{28}$ thus highlighting the importance of PI3K inhibitors in advanced GIST.

Imatinib is approved as a first-line therapy for the treatment of unresectable and/or metastatic GIST, and has shown a PFS of up to 24 months and an overall survival of up to 57 months. ${ }^{29,30}$ Adjuvant imatinib has also shown clinical benefits, including a significant improvement in recurrence-free survival, and $400 \mathrm{mg}$ of imatinib is also approved as adjuvant therapy. ${ }^{10,31}$ Rechallenge with imatinib in patients with metastatic and/or unresectable GIST following objective progression after treatment failure with imatinib and sunitinib has shown encouraging results. The RIGHT trial randomised patients with progressive disease following imatinib/sunitinib with or without other agents, to receive either imatinib rechallenge or placebo (ClinicalTrials.gov, ID No. NCT01151852). ${ }^{32}$ This trial reported a median PFS of 1.8 months in the imatinib rechallenge arm and 0.9 months in the placebo arm. The BFR14 study conducted by the French Sarcoma group also demonstrated the efficacy of imatinib rechallenge in patients who progressed after imatinib discontinuation, and more than $90 \%$ of the patients achieved a tumour response. ${ }^{33}$ In another retrospective trial conducted in Italy in patients with advanced GIST who were previously treated with imatinib, sunitinib and regorafenib, rechallenge with imatinib resulted in a median time to progression of 5.4 months. ${ }^{34}$ This further suggested that rechallenge with imatinib could be an option for patients with advanced GIST. The current study demonstrated an estimated median PFS of 3.5 months $(95 \% \mathrm{Cl}, 1.9-5.4)$ with the combination of buparlisib and imatinib. The results of this Phase 1 trial of buparlisib and imatinib compare favourably with those of the RIGHT trial, although a direct comparison in these small groups is not possible. ${ }^{32}$

Regorafenib is an oral multi-targeted inhibitor with activity against multiple kinases, including KIT, RET, RAF1 and BRAF, as well as those involved in angiogenesis (VEGFR and TIE-2) and in tumour support from the microenvironment (PDGFR and FGFR). ${ }^{35}$ The GRID study, a randomised, Phase 3 trial with regorafenib monotherapy in progressive GIST, reported a median PFS of 4.8 months (hazard ratio, $0.27 ; 95 \% \mathrm{Cl}, 0.19-0.39 ; P<0.0001)$ when treated with the active compound in the third line. ${ }^{36}$ Regorafenib was shown to significantly improve PFS and DCR compared with placebo. In an earlier Phase 2 trial with 34 patients, treatment with third-line regorafenib showed a PFS of 10 months. ${ }^{37}$ The current study and both the GRID and RIGHT studies included patients with advanced GIST who had progressed after failure of at least imatinib and sunitinib. $^{32,36}$ Another study of imatinib in combination with everolimus (targeting the $\mathrm{PI} 3 \mathrm{~K} / \mathrm{AKT} / \mathrm{mTOR}$ pathway) reported a median PFS of 3.5 months $(95 \% \mathrm{Cl}, 1.9-5.2)$ in patients who failed imatinib and sunitinib therapies. ${ }^{38}$ Although preclinical studies suggested that the combination of a PI3K inhibitor with imatinib may be beneficial for these patients, ${ }^{20}$ in this study, buparlisib demonstrated no objective responses and limited DCR with a median treatment exposure of 2.2 months, in combination with imatinib, and a few patients had durable SD. The results from this study may also need to be assessed, considering the relatively good Eastern Cooperative Oncology Group (ECOG) performance status (98\% with ECOG 0 or 1) and slightly lower median age (56.5 years) of the patients enrolled into this study compared with the other studies mentioned, and to patients treated in clinical practice. ${ }^{9,32,34,39}$ Although the study included patients with an ECOG performance status between 0 and 2 considering other criteria for inclusion in the study, it is possible that more patients with a better performance status were included. Although the median age was slightly lower at 56.5 years than that of the other studies, which was mostly around 60 years, meaningful comparisons cannot be drawn between populations across clinical trials as the population age range is overlapping. ${ }^{9,32,34,39}$ Hence, there remains a high unmet medical need in the management of advanced GIST, as highlighted by a median PFS of 4.8 months for regorafenib in the randomised Phase 3 trial, indicating that durable clinical benefit to later lines of therapy remains elusive. ${ }^{36}$

For metastatic GIST, imatinib was used in doses ranging from 400 to $800 \mathrm{mg}$, and a higher PFS was observed with the $800-\mathrm{mg}$ dose in GIST with KIT exon 9 mutations, although the number of interruptions were more. ${ }^{30,40}$ In a Phase 1, dose-finding study of buparlisib in patients with advanced solid tumours, the MTD was determined to be $100 \mathrm{mg} .^{41}$ In this study, the MTD was $80 \mathrm{mg}$ for buparlisib in combination with $400 \mathrm{mg}$ for imatinib, which was $80 \%$ of the MTD of buparlisib as monotherapy (confirmed in Phase 1 studies in patients with solid tumours).

Buparlisib has a toxicity profile, which although not negligible, is manageable, with AEs mostly related to gastrointestinal disorders, hyperglycaemia and mood disorders. ${ }^{42}$ In combination with imatinib, the safety profile was consistent but manageable. As compared with the current standard of care regorafenib, in the GRID trials, the severity of the toxicities was similar as almost $98.5 \%$ of the patients treated with regorafenib experienced an $\mathrm{AE}$ of which $58.3 \%$ experienced an AE of grade 3 and above, although the specific toxicities of the two molecules were different. ${ }^{36}$ Hypertension and hand-foot skin reaction were the most common drug-related AEs with regorafenib of whom $\sim 20 \%$ of the patients experienced grade 3 AEs. The neuropsychiatric AEs were consistent with the previous reports, and could be attributed to the high blood-brain barrier-penetration properties of buparlisib. ${ }^{19,43}$ In the preclinical studies in rodents, buparlisib was able to penetrate the blood-brain barrier and subsequently downregulate the PI3K pathway in the brain of animals.

Although the current study demonstrated slightly better PFS than prior reports of imatinib rechallenge in a resistant population, there was no clear efficacy signal to expect improved clinical outcomes in the context of currently approved therapies for GIST. ${ }^{44}$ Given the limited activity, the benefit-risk balance does not favour the use of this combination in patients who have failed prior therapy with imatinib and sunitinib. Further development of this combination therapy of buparlisib with $400 \mathrm{mg}$ of imatinib is not recommended in the third-line/fourth-line treatment of patients with advanced/metastatic GIST. It would be, however, interesting to further explore this pathway to identify predictors of tumour control under such combination.

\section{ACKNOWLEDGEMENTS}

We thank the patients and their families, investigators and staff from all the participating sites. We thank Amrita Dutta, PhD, for providing medical editorial assistance with this paper.

\section{AUTHOR CONTRIBUTIONS}

H.G. and P.S. contributed to the design of the study, enrolled patients and acquired, analysed and interpreted data. R.L.J., S.G., C.V.M., C.B., J.Y.B., D.J.R., T.D., A.L.C. and M.L. enrolled patients, and acquired and interpreted data. S.B. supervised the trial, and provided support in data acquisition, development of trial database and interpretation of the results. U.B. provided statistical support, and analysed and interpreted the results. P.A. analysed and interpreted the results. All authors have critically reviewed the paper, provided feedback and approved the final version of the paper.

\section{ADDITIONAL INFORMATION}

Ethics approval and consent to participate The study was performed in accordance with the Declaration of Helsinki, and has been reviewed and approved by the independent ethics committee and/or local review board at each participating 
institution (details are provided in Supplementary material). All participants provided written informed consent prior to the study. The study was registered at clinicaltrials. gov (NCT01468688).

\section{Consent to publish Not applicable.}

Data availability Novartis will not provide access to patient-level data, if there is a reasonable likelihood that individual patients could be re-identified. Phase 1 studies, by their nature, present a high risk of patient re-identification; therefore, patient individual results for Phase 1 studies cannot be shared. In addition, clinical data, in some cases, have been collected subject to contractual or consent provisions that prohibit transfer to third parties. Such restrictions may preclude granting access under these provisions. Where co-development agreements or other legal restrictions prevent companies from sharing particular data, companies will work with qualified requestors to provide summary information where possible.

Competing interests All the investigators received a research grant to conduct the trial discussed in the publication. In addition, H.G. reports grants to his institute. R.L.J. received honoraria and consultation fees from Adaptimmune, Blueprint, Clinigen, Eisai, Epizyme, Daichii Sankyo, Deciphera Pharmacuticals, Immunedesign, Johnson and Johnson, Eli Lilly, Merck, Pfizer and Pharmamar. S.G. reports being a consultant and on the advisory board of ARIAD, Pfizer, Bayer, Blueprint Medicines, Deciphera Pharmaceuticals, Exelixis, Eli Lilly, AstraZeneca, Research to Practice, MORE Health and Huron Consulting; reports receiving research funds to her institution from ARIAD, Pfizer, Bayer, Blueprint Medicines, Deciphera Pharmaceuticals and Novartis; royalties from Wolters Kluwer Health (https://www.uptodate.com/home); equity from AbbVie (none currently), Abbott Labs and Allergan; reports holding leadership positions as Vice-Chair Alliance for Clinical Trials in Oncology and Vice-President of Alliance Foundation. CVM reports receiving fees for the advisory board from Novartis, Pfizer and Bayer. J.Y.B. reports receiving grant, personal fees and nonfinancial support from Novartis. D.J.R., M.L. and P. $S$. received institutional funding from Novartis for clinical and translational research in GIST and other sarcomas. T.D. reports research grant and personal fees from Lilly, Chugai Pharma, Kyowa Hakko Kirin, MSD, Daichi Sankyo, Sumitomo Group and Taiho, research grant from Novartis, Merck Serono, Astellas Pharma, Janssen, Boehringer Ingelheim, Takeda, Pfizer, Celgene, Bristol Myers Squibb, AbbVie and Quintiles and personal fees from Amgen outside the submitted work. A.L.C. reports receiving personal fees from Pfizer, Pharmamar, Novartis, Amgen and Lilly. C.B. has no conflicts to disclose. S.H., P.A. and U.B. are employees of Novartis Pharma AG, Basel, Switzerland.

Funding information The study was funded by Novartis Pharma AG, Basel, Switzerland.

Supplementary information is available for this paper at https://doi.org/10.1038/ s41416-020-0769-y.

Note This work is published under the standard license to publish agreement. After 12 months the work will become freely available and the license terms will switch to a Creative Commons Attribution 4.0 International (CC BY 4.0).

Publisher's note Springer Nature remains neutral with regard to jurisdictional claims in published maps and institutional affiliations.

\section{REFERENCES}

1. Ray-Coquard, I., Cassier, P., Labidi Galy, I., Heudel, P., Tassy, L., Chelghoum, M. et al. Combination therapy for gastrointestinal stromal tumors: evidence from recent clinical trials. Clin. Invest 1, 825-836 (2011).

2. Cassier, P. A., Ducimetiere, F., Lurkin, A., Ranchere-Vince, D., Scoazec, J. Y., Bringuier, P. P. et al. A prospective epidemiological study of new incident GISTs during two consecutive years in Rhone Alpes region: incidence and molecular distribution of GIST in a European region. Br. J. Cancer 103, 165-170 (2010).

3. Nilsson, B., Bumming, P., Meis-Kindblom, J. M., Oden, A., Dortok, A., Gustavsson, B. et al. Gastrointestinal stromal tumors: the incidence, prevalence, clinical course, and prognostication in the preimatinib mesylate era-a population-based study in western Sweden. Cancer 103, 821-829 (2005).

4. Corless, C. L. \& Heinrich, M. C. Molecular pathobiology of gastrointestinal stromal sarcomas. Annu Rev. Pathol. 3, 557-586 (2008).

5. Corless, C. L., McGreevey, L., Haley, A., Town, A. \& Heinrich, M. C. KIT mutations are common in incidental gastrointestinal stromal tumors one centimeter or less in size. Am. J. Pathol. 160, 1567-1572 (2002).

6. Corless, C. L., Schroeder, A., Griffith, D., Town, A., McGreevey, L., Harrell, P. et al. PDGFRA mutations in gastrointestinal stromal tumors: frequency, spectrum and in vitro sensitivity to imatinib. J. Clin. Oncol. 23, 5357-5364 (2005).
7. DeMatteo, R. P., Lewis, J. J., Leung, D., Mudan, S. S., Woodruff, J. M. \& Brennan, M. F. Two hundred gastrointestinal stromal tumors: recurrence patterns and prognostic factors for survival. Ann. Surg. 231, 51-58 (2000).

8. Mazzocca, A., Napolitano, A., Silletta, M., Spalato Ceruso, M., Santini, D., Tonini, G. et al. New frontiers in the medical management of gastrointestinal stromal tumours. Ther Adv. Med. Oncol. https://doi.org/10.1177/1758835919841946 (2019).

9. Cohen, M. H., Johnson, J. R., Justice, R. \& Pazdur, R. Approval summary: imatinib mesylate for one or three years in the adjuvant treatment of gastrointestinal stromal tumors. Oncologist 17, 992-997 (2012).

10. Demetri, G. D., van Oosterom, A. T., Garrett, C. R., Blackstein, M. E., Shah, M. H., Verweij, J. et al. Efficacy and safety of sunitinib in patients with advanced gastrointestinal stromal tumour after failure of imatinib: a randomised controlled trial. Lancet 368, 1329-1338 (2006).

11. Mei, L., Du, W., Idowu, M., von Mehren, M. \& Boikos, S. A. Advances and challenges on management of gastrointestinal stromal tumors. Front Oncol. 8, 135 (2018).

12. Demetri, G. D., von Mehren, M., Blanke, C. D., Van den Abbeele, A. D., Eisenberg, B., Roberts, P. J. et al. Efficacy and safety of imatinib mesylate in advanced gastrointestinal stromal tumors. N. Engl. J. Med 347, 472-480 (2002).

13. Serrano, C. \& George, S. Recent advances in the treatment of gastrointestinal stromal tumors. Ther. Adv. Med Oncol. 6, 115-127 (2014).

14. ESMO / European Sarcoma Network Working Group. Gastrointestinal stromal tumors: ESMO Clinical Practice Guidelines for diagnosis, treatment and follow-up. Ann. Oncol. 23(Suppl 7), vii49-vii55 (2012).

15. Kang, Y. K., Kang, H. J., Kim, K. M., Sohn, T., Choi, D., Ryu, M. H. et al. Clinical practice guideline for accurate diagnosis and effective treatment of gastrointestinal stromal tumor in Korea. Cancer Res. Treat. 44, 85-96 (2012).

16. Bauer, S., Duensing, A., Demetri, G. D. \& Fletcher, J. A. KIT oncogenic signaling mechanisms in imatinib-resistant gastrointestinal stromal tumor: PI3-kinase/AKT is a crucial survival pathway. Oncogene 26, 7560-7568 (2007).

17. Duensing, A., Heinrich, M. C., Fletcher, C. D. \& Fletcher, J. A. Biology of gastrointestinal stromal tumors: KIT mutations and beyond. Cancer Invest 22, 106-116 (2004).

18. Maira, S. M., Pecchi, S., Huang, A., Burger, M., Knapp, M., Sterker, D. et al. Identification and characterization of NVP-BKM120, an orally available pan-class I PI3kinase inhibitor. Mol. Cancer Ther. 11, 317-328 (2012).

19. Maira, M., Schnell, C., Lollini, P., Chouaid, C., Schmid, P., Nanni, P. et al. Preclinical and preliminary clinical activity of NVP-BKM120, an oral pan-class I PI3K inhibitor, in the brain. Ann. Oncol. 23(suppl 9), ix537 (2012).

20. Van, Looy, T., Wozniak, A., Floris, G., Sciot, R., Li, H., Wellens, J. et al. Phosphoinositide 3-kinase inhibitors combined with imatinib in patient-derived xenograft models of gastrointestinal stromal tumors: rationale and efficacy. Clin. Cancer Res 20, 6071-6082 (2014).

21. Spitzer, R. L., Kroenke, K., Williams, J. B. \& Lowe, B. A brief measure for assessing generalized anxiety disorder: the GAD-7. Arch. Intern Med 166, 1092-1097 (2006).

22. Kroenke, K., Spitzer, R. L. \& Williams, J. B. The PHQ-9: validity of a brief depression severity measure. J. Gen. Intern Med 16, 606-613 (2001).

23. Wozniak, A., Gebreyohannes, Y. K., Debiec-Rychter, M. \& Schoffski, P. New targets and therapies for gastrointestinal stromal tumors. Expert Rev. Anticancer Ther. 17, 1117-1129 (2017).

24. Quattrone, A., Wozniak, A., Dewaele, B., Floris, G., Vanspauwen, V., Van Looy, T. et al. Frequent mono-allelic loss associated with deficient PTEN expression in imatinib-resistant gastrointestinal stromal tumors. Mod. Pathol. 27, 1510-1520 (2014).

25. Floris, G., Wozniak, A., Sciot, R., Li, H., Friedman, L., Van Looy, T. et al. A potent combination of the novel PI3K Inhibitor, GDC-0941, with imatinib in gastrointestinal stromal tumor xenografts: long-lasting responses after treatment withdrawal. Clin. Cancer Res 19, 620-630 (2013).

26. Bosbach B., Rossi F., Yozgat Y., Loo J., Zhang J. Q., Berrozpe G. et al. Direct engagement of the PI3K pathway by mutant KIT dominates oncogenic signaling in gastrointestinal stromal tumor. Proc. Natl Acad. Sci. USA 114, E8448-E8457 (2017).

27. Massacesi, C., Di Tomaso, E., Urban, P., Germa, C., Quadt, C., Trandafir, L. et al. PI3K inhibitors as new cancer therapeutics: implications for clinical trial design. Onco Targets Ther. 9, 203-210 (2016).

28. Zook, P., Pathak, H. B., Belinsky, M. G., Gersz, L., Devarajan, K., Zhou, Y. et al. Combination of imatinib mesylate and AKT inhibitor provides synergistic effects in preclinical study of gastrointestinal stromal tumor. Clin. Cancer Res 23, 171-180 (2017).

29. Verweij, J., Casali, P. G., Zalcberg, J., LeCesne, A., Reichardt, P., Blay, J. Y. et al. Progression-free survival in gastrointestinal stromal tumours with high-dose imatinib: randomised trial. Lancet 364, 1127-1134 (2004).

30. Blanke, C. D., Rankin, C., Demetri, G. D., Ryan, C. W., von Mehren, M., Benjamin, R. S. et al. Phase III randomized, intergroup trial assessing imatinib mesylate at two dose levels in patients with unresectable or metastatic gastrointestinal stromal 
tumors expressing the kit receptor tyrosine kinase: S0033. J. Clin. Oncol. 26, 626-632 (2008).

31. Dematteo, R. P., Ballman, K. V., Antonescu, C. R., Maki, R. G., Pisters, P. W., Demetri, G. D. et al. Adjuvant imatinib mesylate after resection of localised, primary gastrointestinal stromal tumour: a randomised, double-blind, placebo-controlled trial. Lancet 373, 1097-1104 (2009).

32. Kang, Y. K., Ryu, M. H., Yoo, C., Ryoo, B. Y., Kim, H. J., Lee, J. J. et al. Resumption of imatinib to control metastatic or unresectable gastrointestinal stromal tumours after failure of imatinib and sunitinib (RIGHT): a randomised, placebo-controlled, phase 3 trial. Lancet Oncol. 14, 1175-1182 (2013).

33. Patrikidou, A., Chabaud, S., Ray-Coquard, I., Bui, B. N., Adenis, A., Rios, M. et al. Influence of imatinib interruption and rechallenge on the residual disease in patients with advanced GIST: results of the BFR14 prospective French Sarcoma Group randomised, phase III trial. Ann. Oncol. 24, 1087-1093 (2013).

34. Vincenzi B., Nannini M., Badalamenti G., Grignani G., Fumagalli E., Gasperoni S. et al. Imatinib rechallenge in patients with advanced gastrointestinal stromal tumors following progression with imatinib, sunitinib and regorafenib. Ther. Adv. Med. Oncol. https://doi.org/10.1177/1758835918794623 (2018).

35. Rutkowski, P. \& Stepniak, J. The safety of regorafenib for the treatment of gastrointestinal stromal tumors. Expert Opin. Drug Saf. 15, 105-116 (2016).

36. Demetri, G. D., Reichardt, P., Kang, Y. K., Blay, J. Y., Rutkowski, P., Gelderblom, H. et al. Efficacy and safety of regorafenib for advanced gastrointestinal stromal tumours after failure of imatinib and sunitinib (GRID): an international, multicentre, randomised, placebo-controlled, phase 3 trial. Lancet 381, 295-302 (2013).

37. George, S., Wang, Q., Heinrich, M. C., Corless, C. L., Zhu, M., Butrynski, J. E. et al. Efficacy and safety of regorafenib in patients with metastatic and/or unresectable
GI stromal tumor after failure of imatinib and sunitinib: a multicenter phase II trial. J. Clin. Oncol. 30, 2401-2407 (2012).

38. Schoffski, P., Reichardt, P., Blay, J. Y., Dumez, H., Morgan, J. A., Ray-Coquard, I. et al. A phase I-II study of everolimus (RAD001) in combination with imatinib in patients with imatinib-resistant gastrointestinal stromal tumors. Ann. Oncol. 21, 1990-1998 (2010).

39. Montemurro, M., Gelderblom, H., Bitz, U., Schutte, J., Blay, J. Y., Joensuu, H. et al. Sorafenib as third- or fourth-line treatment of advanced gastrointestinal stromal tumour and pretreatment including both imatinib and sunitinib, and nilotinib: a retrospective analysis. Eur. J. Cancer 49, 1027-1031 (2013).

40. Blanke, C. D., Demetri, G. D., von Mehren, M., Heinrich, M. C., Eisenberg, B., Fletcher, J. A. et al. Long-term results from a randomized phase II trial of standard- versus higher-dose imatinib mesylate for patients with unresectable or metastatic gastrointestinal stromal tumors expressing KIT. J. Clin. Oncol. 26, 620-625 (2008).

41. Bendell, J. C., Rodon, J., Burris, H. A., de Jonge, M., Verweij, J., Birle, D. et al. Phase I, dose-escalation study of BKM120, an oral pan-Class I PI3K inhibitor, in patients with advanced solid tumors. J. Clin. Oncol. 30, 282-290 (2012).

42. Patsouris, A., Augereau, P., Frenel, J. S., Robert, M., Gourmelon, C., Bourbouloux, E. et al. Benefits versus risk profile of buparlisib for the treatment of breast cancer. Expert Opin. Drug Saf. 18, 553-562 (2019).

43. Nanni, P., Nicoletti, G., Palladini, A., Croci, S., Murgo, A., lanzano, M. L. et al. Multiorgan metastasis of human HER-2+ breast cancer in Rag2-/-;Il2rg-/- mice and treatment with PI3K inhibitor. PLOS ONE 7, e39626 (2012).

44. Blay, J. Y., Perol, D. \& Le Cesne, A. Imatinib rechallenge in patients with advanced gastrointestinal stromal tumors. Ann. Oncol. 23, 1659-1665 (2012). 